\title{
MÁS ALLÁ DEL APRENDIZAJE ESCOLAR: EL ROL DE LA SUBJETIVIDAD EN EL ENFOQUE DE LOS FONDOS DE IDENTIDAD
}

\author{
Overcoming school learning: The role of the subjectivity in \\ the funds of identity approach \\ David SUBERO, Moisés ESTEBAN-GUITART \\ Universidad de Girona. España. \\ david.subero@udg.edu; moises.esteban@udg.edu \\ bttps://orcid.org/0213-2362-4737-0047; bttps://orcid.org/0213-2362-1700-8792
}

Fecha de recepción: 22/06/2019

Fecha de aceptación: 17/10/2019

Fecha de publicación en línea: 01/01/2020

\section{RESUMEN}

El aprendizaje ocurre cuando el aprendiz se convierte en sujeto de su propio aprendizaje en contextos tanto formales como informales. La teoría de la subjetividad de González Rey, que parte del enfoque histórico-cultural, afirma que dicho proceso está guiado por la emoción, la imaginación y la creatividad, que a su vez promueve el desarrollo subjetivo. Sin embargo, las actividades de aprendizaje tradicionales han ignorado al aprendiz como sujeto. Los proyectos basados en fondos de identidad favorecen el desarrollo de nuevas formas de reconocer al aprendiz fomentando nuevos modos de intercambiar experiencias de aprendizaje dentro y fuera de la escuela. Los fondos de identidad se erigen de este modo como espacios en donde se reconocen intereses, aficiones, experiencias y vivencias por parte de los jóvenes como recursos importantes para estimular su desempeño académico. Específicamente, la subjetividad permite que el aprendiz se convierta en sujeto a partir de la producción de sus propios procesos de subjetivación mediante la práctica dialógica y comprensiva. El objetivo principal de este artículo es ir un paso más allá en relación con los fondos de identidad ilustrando una serie de experiencias educativas que ayudan a identificar y movilizar dichos fondos mediante el desarrollo de la subjetividad. Estos fondos de identidad subjetivados son integrales para los educadores con el objeto de favorecer 
posicionamientos reflexivos en los alumnos que encaminen hacia la generación de nuevos procesos de subjetivación. Vehicular espacios de encuentro entre los fondos de identidad y la subjetividad se sugiere una forma pertinente para avanzar hacia mayores cotas de significación y sentido por parte de los jóvenes en la educación del siglo XXI.

Palabras clave: aprendizaje; psicología educativa; identidad; experiencia emocional; educación cultural; inclusión.

\section{ABSTRACT}

Learning occurs when the learner becomes the subject of his own learning in both formal and informal contexts. The subjectivity theory of González Rey, which starts from a historical-cultural approach, affirms that this process is guided by emotion, imagination and creativity, which in turn promotes subjective development. However, traditional learning activities have ignored the apprentice as a subject. Projects based on funds of identity favor the development of new ways to recognize the learner by encouraging new ways of exchanging learning experiences inside and outside the school. Identity funds are thus built as spaces where interests, hobbies and experiences are recognized by young people as important resources to stimulate their academic performance. Specifically, subjectivity allows the learner to become a subject from the production of his own processes of subjectivation through dialogic and comprehensive practice. The main objective of this article is to go one step further in relation to identity funds by illustrating a series of educational experiences that help identify and mobilize these funds through the development of subjectivity. These subjective identity funds are integral to educators in order to favor reflective positions in students who are moving towards the generation of new processes of subjectivation. Lead meeting spaces between identity funds and subjectivity suggest a relevant way to move towards higher levels of significance and meaning on the part of young people in 21st century education.

Keywords: learning; educational psychology; identity; emotional experience; cultural education; inclusion.

\section{INTRODUCCIÓN}

Existe un amplio consenso sobre cómo la educación en el siglo XXI ha de dirigirse hacia formas prospectivas de aprendizaje con el objetivo que las personas creen y desarrollen nuevos conocimientos y comprensiones de forma colaborativa, así como el importante papel que ejercen los entornos escolares, extraescolares, familiares y comunitarios en este desempeño (Livingstone y Sefton-Green, 2016; Erstad y Sefton-Green, 2012).

En este sentido, Alex Kozulin (1998) distinguió dos tipologías de educación; la retrospectiva y la prospectiva. La primera responde al objetivo social de transmitir 
una tradición cultural sólida e incuestionable de generación en generación. Es decir, «el modelo del mundo y la tradición cultural están dados, y la tarea de un estudiante es absorber esta tradición y las herramientas intelectuales asociadas con ella» (p. 154).

La educación retrospectiva empata con dos perspectivas que han sido centrales en las teorías del aprendizaje. Ambas concepciones parten de una visión tradicional y hegemónica en educación en donde se entiende el aprendizaje bajo una visión 1) lógico-cognitiva (Saz, Engel y Coll, 2016) y 2) práctico-asimilativa (Talizina, 2000; Williams, 1999). La visión lógico-cognitiva, se le atribuye una preponderancia de los aspectos lógicos, cognitivos e intelectuales sobre el carácter subjetivo del estudiante, su emocionalidad, así como en las formas singulares de implicación subjetiva con relación al aprendizaje. La visión práctico-asimilativa, destaca la relación directa entre el individuo y el objeto que va a ser aprendido. Como la realidad externa está dada y estable, es necesario internalizarla mediante la actividad con objetos materiales por parte del aprendiz. El proceso de internalización de lo externo a lo interno conforma el proceso de aprendizaje. Así, el aprendizaje es un proceso que tiene su base en las operaciones prácticas con los objetos externos de tal modo que el pensamiento es un epifenómeno de la actividad práctica (Talizina, 2000; Talizina, Solovieva, Quintanar-Rojas, 2010). De este modo, las actividades educativas promueven que el adulto medie con relación a los aprendices a través de acciones prácticas con los objetos. Así, el aprendizaje y la enseñanza se reducen a prácticas estandarizadas y técnicas instrumentales que responden sobre "qué hacer» y "cómo hacerlo» y «durante cuánto tiempo hacerlo» (Madeira-Coelho y Tacca, 2019, p. 87).

Sin embargo, esta forma de entender la educación ha quedado desfasada, ya que los jóvenes de hoy tienen a su alcance formas inimaginables de aprender hace apenas una generación. En una sociedad interconectada, el aprendizaje se vincula más fácilmente con contextos diversificados a través de las oportunidades que ofrecen las nuevas tecnologías. A través de los medios digitales y las nuevas formas de relación y comunicación se generan innumerables oportunidades accesibles para compartir, crear y expandir el conocimiento. Es decir, hoy en día el aprendizaje posibilita ser participantes, creadores y personas comprometidas en una investigación activa y autodirigida; lo que se ha venido a llamar aprendizaje interconectado (Ito et al., 2013).

A raíz de estas nuevas necesidades en el aprendizaje, la propuesta de aprendizaje prospectivo plantea que los aprendices han de ser capaces de afrontar problemas todavía inexistentes. Al hacerlo, el estudiante se dirige hacia la producción en vez de hacia la reproducción del conocimiento. Lo relevante de esta propuesta es su incidencia en que el conocimiento no aparece de forma sólida en resultados y soluciones, sino que emerge a partir de un proceso creativo, el proceso de «autoría» (Kozulin, 1998, p. 154).

Acorde con esta perspectiva, el aprendizaje no se conceptualiza como un proceso cognitivo que se desarrolla en la mente de forma individual. Es decir, la 
persona no aprende solamente con el intelecto o a través de la participación en diferentes prácticas sociales. Una propuesta coherente con lo anterior es la manera de comprender el aprendizaje por parte de la teoría de la subjetividad de corte histórico-cultural elaborada por González Rey en donde se entiende éste como un sistema complejo, articulado en forma de historias de vida del aprendiz a través de sus experiencias en los diversos y diferentes contextos en donde transita (González Rey, 2014a). La emoción, y específicamente las unidades simbólicoemocionales permiten el desarrollo de procesos subjetivos singulares en el aprendiz que incluyen prácticas dialógicas, comunicativas y reflexivas (González Rey, 2018). Las emociones históricamente han sido relegadas del estudio de la psique o bien entendidas como subproductos de la cognición. Con la unidad entre lo simbólico y lo emocional, las emociones se convierten en unidades dinámicas que posibilitan a éstas trascender la regulación natural y adquirir una nueva cualidad psíquica, su relación inseparable con el pensamiento y el lenguaje, lo que las hace inseparables del desarrollo cultural del hombre (González Rey, 2011). Lo característico de los aprendizajes guiados por las unidades simbólico-emocionales es que permiten a los individuos transformar el ambiente y a ellos mismos, contra argumentando los enfoques psicológicos que entienden a la persona en un proceso de adaptación. De este modo, la forma en cómo el aprendiz, en su contexto, produce una acción singular a partir de la personalización de una información dada y la producción de ideas nuevas es coherente con el desarrollo de un proceso de aprendizaje creativo, fundamentado en la subjetividad del alumno (Mitjáns Martínez, 2013a). Este tipo de aprendizaje ejerce un mayor impacto y posibilita el desarrollo de habilidades críticas en el alumno, así como la motivación en crear e inventar nuevas soluciones para diferentes situaciones de vida (Madeira-Coelho y Tacca, 2019).

Hay dos ideas para tener en cuenta a la hora de conceptualizar el aprendizaje en una sociedad interconectada. Una proviene del concepto de aprendizaje prospectivo que afirma que el aprendizaje emerge a partir de procesos de creatividad, de construcción y de autoría genuina por parte del alumno caracterizado por la producción que el aprendiz hace y por los procesos subjetivados en ella implicados (Mitjáns Martínez, 2013a). Además, el aprendizaje implica tener en cuenta las trayectorias y experiencias incrustadas en el aprendizaje prospectivo y creativo. En otras palabras, con la globalización, para adaptarse a los cambios de un mundo global e interconectado, será cada vez más necesario un trabajo creativo en donde el sistema educativo actual deba formar estudiantes que sean capaces de movilizar habilidades de pensamiento crítico, innovador y creativo que serán necesarias en la economía global (Araya y Michael, 2010; National Research Council, 2012).

En segundo lugar, ponemos la atención en el aprendizaje experiencial y contextualizado (Luning, Wyatt y Im, 2011). El aprendizaje ocurre de una forma más atractiva y exitosa cuando los procesos de diálogo y comunicación incluyen experiencias de vida de los estudiantes en ambientes informales tales como los de su 
hogar, comunidad o entornos virtuales. El modelo CREDE (2004) indicó una mayor participación y aprendizaje cuando los profesores se acercaban a las experiencias de vida y aprendizaje de los estudiantes. La instrucción, si quiere ser coherente con el desarrollo de una pedagogía efectiva, tiene que facilitar la conexión de las experiencias de conocimiento y aprendizaje de los alumnos con el conocimiento académico y las experiencias de aprendizaje escolar mediante la transformación, la generación de alternativas y la propuesta de nuevas experiencias y prácticas pedagógicas (Esteban-Guitart, 2016; Esteban-Guitart y Moll, 2014a).

Los aprendizajes que reconocen las experiencias de vida de los estudiantes fuera de la escuela y los vehiculan hacia la institución escolar, han sido a menudo ignorados, no reconocidos o incluso prohibidos por las prácticas dominantes en la educación formal. Es decir, las necesidades, intereses, placeres o curiosidades de los aprendices no han tenido cabida en la escuela, de forma generalizada (Subero, Vujasinović y Esteban-Guitart, 2017). Tenerlos en cuenta, puede facilitar procesos de enseñanza y aprendizaje creativos, dinámicos y vivenciales siendo una demanda para el incremento de las competencias de los niños y los jóvenes en el aprendizaje, especialmente para aquellos grupos que tienen una mayor dificultad en ser reconocidos en las prácticas académicas por sus bajos ingresos, grupo étnico minoritario o bien de origen extranjero. Es decir, los alumnos «infra-representados» (Rios-Aguilar et al., 2011 p. 179).

Los fondos de conocimiento (González, Moll y Amanti, 2005) han sido una de las propuestas educativas que ha intentado superar la dicotomía dentro y fuera con relación al aprendizaje; fundamentados en un enfoque sociocultural. En particular, «los fondos de conocimiento parten de la premisa que las familias cuentan con redes sociales y de intercambio en su vida cotidiana que resultan esenciales para el bienestar del núcleo familiar» (Moll, 2014 p. 25). Desde esta perspectiva el aprendizaje es entendido a través de la mediación. Es decir, la persona se entiende como participante en diversas prácticas culturales a través de otras personas o diversos artefactos, enfatizando el carácter transaccional del aprendizaje humano (Moll, 2014). De este modo, el conocimiento no se reduce a propiedades individuales sino que está distribuido entre las personas, artefactos, actividades y dispositivos en donde el alumno participa. Lo relevante en este proceso son las transacciones sociales y las actividades culturales que se movilizan para conectar las experiencias y conocimientos familiares de los alumnos con las prácticas escolares.

Por una parte, los fondos de conocimiento son pertinentes para superar las visiones culturalmente deficitarias con relación a las familias (Esteban-Guitart y Vila, 2013). Desde la teoría del déficit se pone el énfasis en que los estudiantes procedentes de minorías étnicas y culturales traen consigo una suerte de carencias, dificultades y demás impedimentos que les imposibilitan rendir satisfactoriamente en las escuelas. Los fondos de conocimiento entienden a las familias como poseedoras de cuerpos de conocimientos susceptibles de ser reconocidos y validados 
por la institución escolar en contextos de desarrollo donde el aprendiz participa y adquiere aprendizajes (Gonzalez, Moll y Amanti, 2005). Sin embargo, con relación al aprendizaje prospectivo, entendemos que el planteamiento de los fondos de conocimiento no llega a responder al desarrollo de procesos de construcción de conocimiento como creación, sino que se encuentra en un estadio inferior que responde al desarrollo de procesos de construcción de conocimiento vinculados a la participación. Es decir, las prácticas y experiencias familiares que son relevantes para el alumno se entienden necesarias para llevarlas a las prácticas escolares formalizadas. Sin embargo, aparentemente no existe un proceso de aprendizaje en que el alumno sea capaz de superar la analogía entre participación y aprendizaje y dirigirse hacia cotas de desarrollo más genuinas y singulares vinculadas con la creación de conocimiento.

Por su parte, los proyectos basados en los fondos de identidad (Esteban-Guitart, Subero, y Brito-Rivera, 2015; Esteban-Guitart, 2016) reconocen las afiliaciones, aficiones e intereses de los jóvenes como recursos importantes para hacer posible la estimulación académica por parte de los alumnos (Azevedo, 2013). A diferencia de los fondos de conocimiento, son las identidades y las experiencias de los alumnos las que guían la emergencia del conocimiento y promueven el aprendizaje más allá del contexto escolar. La identidad se entiende en términos socioculturales como algo que reside simultáneamente dentro y fuera. Es decir, "la identidad está en las cosas y en las personas y en cómo las personas se auto-expresan, se autoconstruyen y se auto-comprenden a partir de ellas» (Esteban-Guitart y Moll, 2014a, p. 37). Por ello, la lógica de los fondos de identidad sugiere entender como fondos identitarios no solamente los conocimientos familiares, sino todas aquellas experiencias de vida de los aprendices que en última instancia les ayuden a definirse (Subero, Vujasinović y Esteban-Guitart, 2017). Estas experiencias internalizadas son movilizadas por los estudiantes para mediar en la identidad humana y pueden incorporarse y distribuirse en personas, objetos y artefactos (Esteban-Guitart, 2016; Esteban-Guitart y Moll, 2014a).

Sin embargo, sugerimos que para posibilitar aprendizajes que tiendan hacia mayores cotas de creatividad, construcción y de autoría genuina por parte del alumno es necesario situarse en el marco de la teoría de la subjetividad de corte histórico-cultural. Las concepciones teoréticas-conceptuales que integran la teoría de la subjetividad permiten nuevas posibilidades a favor de la emergencia de la singularidad de la persona y avanzan en una mayor cohesión y precisión en los procesos de teoría y práctica, tomando en cuenta las necesidades de la sociedad de innovar en los contextos educacionales (Oliveira Campolina y Mitjáns Martínez, 2016; Madeira-Coelho y Tacca, 2019). Desde la perspectiva de la teoría de la subjetividad, el aprendizaje es una producción subjetiva. Es decir, es un proceso «que se configura a través de sentidos subjetivos que expresan múltiples experiencias socioculturales del aprendiz en un nivel simbólico-emocional» (González Rey y 
Mitjáns Martínez, 2017a, p. 64). De este modo, a partir de unidades auto-generativas, es posible el desarrollo de un aprendizaje dirigido por la fantasía y la imaginación en singular. En el marco de la teoría de la subjetividad, los fondos de identidad pueden maximizar su potencial de aprendizaje configurándose subjetivamente, abriendo nuevas vías de subjetivación en el aprendiz y posibilitando la emergencia del sujeto (Subero, en prensa). Por sujeto entendemos «un individuo o grupo que es capaz de generar un camino alternativo de subjetivación en el espacio institucional normativo en el que actúa” (Mitjans Martínez y González Rey, 2017, p.52). Véase la escuela como institución escolar o cualquier otra institución normativa. Cuando la persona tiene la posibilidad de generar configuraciones subjetivas que le permitan producir nuevos recursos subjetivos para trascender sus circunstancias inmediatas, el individuo se convierte en sujeto de la situación. Es decir, coge las riendas de su subjetividad tomando decisiones de forma activa que son generadas a partir de sus sentidos subjetivos.

En este artículo, usando ejemplos que vienen del marco de la literatura de la teoría de la subjetividad, vamos a discutir y a ilustrar un número de estrategias que pueden ser utilizadas para favorecer el desarrollo de fondos de identidad subjetivados más allá del aprendizaje escolar que sean compatibles con la visión prospectiva del aprendizaje. En otras palabras, vamos a discutir algunas experiencias educativas que pueden favorecer la reflexión y el avance de los fondos de identidad en un marco subjetivo enfatizando el rol relevante del aprendizaje creativo en el proceso.

\section{LOS FONDOS DE IDENTIDAD}

Los fondos de identidad, como hemos introducido más arriba, tienen su origen en el desarrollo del enfoque de los fondos de conocimiento en donde las familias cuentan con «cuerpos de conocimiento culturalmente desarrollados e históricamente acumulados, así como las habilidades esenciales para el funcionamiento y bienestar familiar o individual» (Moll 1997, p. 47). Las prácticas familiares son cuerpos de conocimiento fundamentales para construir en las aulas mejores procesos de desarrollo en los alumnos. Los profesores, acuden a los hogares de los alumnos y establecen interacciones diferentes mediante entrevistas y encuentros con el objetivo de recoger información sobre los fondos de conocimiento familiares. En un segundo término, estos fondos de conocimiento recolectados se reconfiguran y se llevan a cabo en las aulas a partir de prácticas educativas por parte de los docentes (González, Moll y Amanti, 2005).

Sin embargo, el foco de análisis casi exclusivo en las prácticas familiares hace que quede en un segundo plano la creación de los mundos sociales de vida a partir de los propios intereses y experiencias de los aprendices tales como los grupos de amigos, «hobbies» o internet. Es decir, no siempre lo que se hace con la familia tiene que ser relevante en la construcción de los fondos de conocimiento del alumno ya que, quizá, aquello importante para los niños y jóvenes es independiente de la 
vida social de los adultos con los que se relaciona. Por ello, en busca de un aprendizaje encaminado hacia la prospectividad, son necesarias prácticas educativas que enfaticen los talentos, pasiones e intereses de los estudiantes como fuente de sus fondos de conocimiento y práctica escolar (Subero, Vila y Esteban-Guitart, 2015).

Los fondos de identidad parecen ser sensibles con la idea de un aprendizaje prospectivo. Para Esteban-Guitart y Moll (2014a) los fondos de conocimiento se convierten en "fondos de identidad cuando las personas los utilizan para autodefinirse. En particular, entendemos por fondos de identidad los recursos, socialmente distribuidos, históricamente acumulados y culturalmente desarrollados esenciales en la auto-comprensión, auto-expresión y auto-definición. Dicho con otras palabras, la noción de fondos de identidad denota una serie de recursos o caja de signos e instrumentos» (p.37). De este modo, los fondos de identidad posibilitan la auto-definición y auto-conformación de los alumnos a partir de sus propios mundos sociales, trayectorias y experiencias. A través de estas prácticas culturales y experiencias de vida, fundamentadas en los procesos de la vida cotidiana y de las actividades diarias, los individuos internalizan, usan y crean fondos de identidad, es decir, recursos semióticos distribuidos que median la identidad humana. Por ello, la identidad no solamente existe en la mente de los individuos, sino que está distribuida a través de las personas (otros significantes), artefactos, actividades y escenarios (Esteban-Guitart y Moll, 2014b). Desde esta perspectiva, los fondos de identidad incluyen objetos, actividades, o personas que forman parte de nuestra experiencia significativa. Para la recogida de estos fondos se hace uso de la multimetodología autobiográfica extendida (Esteban-Guitart, 2012; Esteban-Guitart, 2016). La multi-metodología autobiográfica extendida o MAE son «un conjunto de técnicas proyectivas donde el alumno se representa mediante un gráfico, un símbolo o un elemento visual simbólico» (p.591). De este modo la finalidad de dicha metodología es que el uso de recursos textuales, gráficos o visuales pueda facilitar el estímulo del proceso de reflexión en el alumno.

Sin embargo, parecen existir algunas dudas en relación con la conformación de los fondos de identidad por parte de los aprendices, ya que el desarrollo de estos fondos no siempre correlaciona con la emergencia de las experiencias de vida en tanto que sentidos simbólicos-emocionales singulares en la persona. Algo que si está presente en la teoría de la subjetividad de corte histórico-cultural.

En primer lugar, entender los fondos de identidad a partir de interacciones sociales entre personas en un contexto dado (Esteban-Guitart y Moll, 2014b), se aleja de la comprensión del desarrollo humano como flujo de unidades simbólicoemocionales que son autogeneradas. Entendemos que el aprendizaje es mucho más que un proceso de comprensión y uso de significados y operaciones culturales que se internalizan y se asimilan, ya que, para los seres humanos, el aprendizaje es inseparable de la subjetividad representando el carácter específicamente humano del desarrollo (González Rey y Mitjáns Martínez, 2017b). Vincular la identidad de 
forma directa a un contexto social dado o a las interacciones sociales de la persona, entendemos que no permite la incorporación de la subjetividad en el proceso de aprender. El aprendizaje como producción subjetiva, va más allá de categorías apriorísticas y predicciones sobre qué acciones desarrollar en la práctica educativa. Es decir, asumiendo que el desarrollo de los recursos subjetivos constitutivos del aprendizaje pasa por el aprendiz, no es posible garantizar el efecto de una acción intencional externa sin tenerlo en cuenta (Mitjáns Martínez, 2013a). De este modo, es necesario ir más allá de una comprensión aparentemente causal de los procesos de enseñanza y aprendizaje sugiriendo producciones subjetivas de la experiencia vivida por parte del alumno como una cualidad específica y singular de los procesos humanos (González Rey y Mitjáns Martínez, 2017a).

En segundo lugar, desde los fondos de identidad se entiende que, a través de las prácticas de aprendizaje, los individuos crean artefactos identitarios que les permiten ampliar sus conocimientos, habilidades y experiencias (Esteban-Guitart y Moll, 2014a). Entendemos que no podemos limitar los procesos de creación de conocimiento e identidad al uso o construcción de artefactos mediadores, al menos no desde una ontología no subjetiva. Es necesario plantear un nuevo marco ontológico subjetivo que vehicule procesos que vayan más allá del uso de artefactos, ya que el flujo de unidades y configuraciones simbólico-emocionales excede las posibilidades de articulación y acumulación de información que posibilitan dichos instrumentos. De este modo, se plantea la necesidad de articular miradas teóricasepistemológicas-metodológicas particulares para dar respuesta a la anterior disyuntiva en donde los procesos dialógicos y comunicacionales sean el foco (González Rey y Mitjáns Martínez, 2017b).

Finalmente, colocar los fondos de identidad de los estudiantes en el centro de la instrucción permite a los conocimientos y experiencias previas del alumno conectarse con nuevos conocimientos académicos y experiencias formales de aprendizaje. Sin embargo, colocar la teoría de la subjetividad en el centro del desarrollo de los fondos de identidad de los estudiantes sugiere una mayor y mejor aproximación a la complejidad y desarrollo de los procesos de aprendizaje de los alumnos, que siempre serán subjetivados y vehiculados directa o indirectamente en forma de metáfora o simbolismo (González Rey, 2018).

De este modo, se sugiere que la emergencia de conocimientos y experiencias de aprendizaje basadas en fondos de identidad precisan de nuevas formas de comprensión de los procesos de enseñanza y aprendizaje en un marco subjetivo. Entendemos que, así, es posible dirigirse hacia un mejor desarrollo del aprendizaje prospectivo basado en la creación de conocimiento avanzando en la atención de las identidades de los estudiantes de forma compleja, holística, constructiva y subjetiva. Es decir, hacia un aprendizaje creativo, donde se personaliza la información dada y se producen ideas nuevas (Mitjáns Martínez, 2013a). 
DAVID SUBERO Y MOISÉS ESTEBAN-GUITART

MÁS ALLÁ DEL APRENDIZAJE ESCOLAR: EL ROL DE LA SUBJETIVIDAD

EN EL ENFOQUE DE LOS FONDOS DE IDENTIDAD

\section{LA IDENTIDAD EN LA TEORÍA DE LA SUBJETIVIDAD}

La teoría de la subjetividad de corte histórico-cultural parte de una premisa sencilla pero fundamental para entender la complejidad del desarrollo psicológico. Es decir, no se entiende la subjetividad como un entramado intrapsíquico, sino que es una producción cualitativamente diferenciada de los seres humanos dentro de las condiciones sociales, culturales e históricamente situadas en las que vivimos (González Rey y Mitjáns Martínez, 2017a). Este entramado subjetivo, pese a tener su origen en la cultura, nunca tienen una relación lineal con el exterior ya que para la teoría de la subjetividad la experiencia vivida es una cualidad específica y singular de los procesos humanos. De este modo, procesos cognitivos tradicionales tales como la inteligencia, la cognición o la psique adquieren una nueva cualidad procesual a través de producciones simbólico-emocionales que tienen una relación irrenunciable en la cultura y en la historia de vida del individuo pero que no se supeditan a ella; estamos hablando de los sentidos subjetivos (González Rey y Mitjáns Martínez, 2017). Los sentidos subjetivos son unidades simbólico-emocionales dinámicas y flexibles que se organizan en el curso de las acciones y experiencias de los individuos en configuraciones subjetivas. La configuración subjetiva es un dispositivo complejo e integral que comprende sentidos subjetivos que han sido subjetivados a partir de las experiencias históricas vividas por la persona. Sin embargo, lo que establece una diferencia fundamental a otras posturas ontológicas histórico-culturales en relación con el proceso de desarrollo es la independencia efectiva que adquiere la persona en abrir nuevas vías de subjetivación, fuera de condicionantes, determinantes o causalidades tanto biológicas como sociales. Estos sentidos subjetivos parten de nuevas unidades simbólico-emocionales que son autogeneradas por el propio sujeto de forma que la imaginación, la fantasía y la creatividad vehiculan su emergencia y desarrollo. De este modo, el desarrollo subjetivo se abre paso a partir de nuevos recursos subjetivos que permiten al individuo hacer cambios cualitativos en diferentes áreas de la vida, generando una implicación personal profunda en el área en que la configuración subjetiva de desarrollo emerge» (González Rey y Mitjáns Martínez, 2017b). Para que el desarrollo subjetivo sea posible, es necesario el cambio de postura del individuo hacia la emergencia como sujeto; ya que la condición de sujeto permite abrirse a nuevos procesos de subjetivación, durante el cual nuevos recursos subjetivos son generados (González Rey y Mitjáns Martínez, 2017b).

Valoremos las implicaciones anteriores con relación al aprendizaje. Si entendemos que el aprendizaje es una producción subjetiva, se sugiere necesario que los procesos que envuelvan el aprendizaje requieran la emergencia del aprendiz como sujeto. Es decir, el aprendizaje ha de posibilitar que el aprendiz —en su condición de sujeto- genere vías alternativas de subjetivación en el espacio institucional normativo en que ellos actúan; en este caso en la escuela (González Rey y Mitjáns Martínez, 2017b). La escuela, así como la familia, los amigos, las nuevas tecnologías o los espacios virtuales de aprendizaje son espacios sociales que tienen una 
incidencia tanto en el desarrollo subjetivo del aprendiz, como en la emergencia del sujeto. Sin embargo, estos espacios sociales no tienen una representación directa en el aprendiz, sino que se configuran subjetivamente a través de sentidos subjetivos de forma singular. De este modo, los diferentes espacios sociales y sus unidades simbólico-emocionales forman parte del flujo de sentidos subjetivos y configuraciones subjetivas del aprendiz. En otras palabras, las configuraciones sociales subjetivas que emergen a través de procesos relacionales e institucionalizados, inciden en el aprendiz representando nuevos recursos de sentidos subjetivos a partir de cada espacio social específico en donde la persona vive su experiencia; es lo que se ha venido a llamar subjetividad social (Mitjáns Martínez y González Rey, 2017). De este modo, las formas singulares que tiene el sujeto de hacer emerger las unidades simbólico-emocionales de origen social a partir de diferentes historias de vida y contextos tales como situaciones familiares de un padre ausente, necesidad de ser aceptado y protegido por los amigos, el bienestar que le genera dibujar con un hermano o bien el sistema relacional de confianza con el profesor son producciones subjetivas que van más allá del espacio social escolar en donde se desarrollan los procesos de enseñanza y aprendizaje formal. Es decir, las subjetividades sociales del alumno trascienden la institución escolar, pero a su vez son indispensables para favorecer los aprendizajes del aprendiz en la escuela, ya que forman parte de la configuración subjetiva del alumno. Este hecho justifica tener muy en cuenta la subjetividad social (además de la individual) para favorecer espacios de desarrollo y producciones subjetivas que fomenten el aprendizaje escolar.

Los fondos de identidad bajo un marco subjetivo de aprendizaje se sugieren como una estrategia favorecedora de espacios de desarrollo y producciones subjetivas. Avanzar en una definición de la identidad como proceso subjetivo supone comprender, no solamente las unidades simbólico-emocionales en una ontología diferente, sino la complejidad del proceso en donde dichas unidades cualitativamente diferentes emergen y se configuran subjetivamente. Para abrir vías de subjetivación reales en la persona, es necesario hacer emerger la figura del sujeto y la subjetividad social en el marco de la teoría de la subjetividad (Subero, en prensa). Se propone de este modo comprender la identidad como una configuración subjetiva que implica una cualidad específica de los sistemas subjetivamente configurados de los aprendices (González Rey, 2014a). De este modo, entendemos pertinente reconfigurar la categoría de identidad como una configuración subjetiva que moviliza el aprendizaje del estudiante.

Tradicionalmente, la identidad se ha entendido como una función u operación que ha sido ampliamente empleada por la psicología en el estudio de las funciones cognitivas. De este modo, se reducía a una categoría individual que se localizaba únicamente dentro de la mente del individuo definida como algo fijo en la cabeza de las personas, siendo un rasgo de su personalidad. Los fondos de identidad clásicos, rompieron con esta visión cognitiva del concepto de identidad y la situaron como 
una categoría que tenía su origen en la cultura, de tal forma que no se podía reducir a propiedades ni rasgos individuales. De esta forma, la identidad estaba distribuida en el exterior y se corporizaba en diferentes personas, artefactos, actividades y contextos en donde el aprendiz interactuaba (Esteban-Guitart y Moll, 2014a; 2014b; Penuel y Wertsch, 1995). En los fondos de identidad, las personas se definen a sí mismas a través de otras personas y a través de los artefactos y recursos de sus mundos sociales y culturales. En ese sentido, las relaciones sociales, otras personas significativas, actividades y prácticas particulares, ideologías políticas, creencias religiosas o cualquier otro artefacto, como «una bandera o una canción, se convierten en recursos para crear y expresar identidad" (Esteban-Guitart y Moll, 2014a, p. 36).

Desde el marco de la subjetividad, la identidad tiene una cualidad subjetiva específica que es intrínseca a la configuración subjetiva de cualquier acción humana. Viendo la identidad como una producción subjetiva, buscamos ir más allá de la lógica positivista y reduccionista en la que la categoría se establece como influencia y atributo determinante en la acción del individuo. La subdivisión de la categoría de identidad en cinco grandes ámbitos temáticos 1) geográficos, 2) prácticos, 3) actividad, 4) sociales, 5) institucionales (Esteban-Guitart y Moll, 2014a) es un buen ejemplo de ello. Superando la lógica categorial, la identidad emerge como producción subjetiva en lugar de ser simplemente otra función u operación ampliamente empleada por la psicología en el estudio de las funciones cognitivas.

La identidad, como cualquier producción subjetiva, es intrínseca a las configuraciones subjetivas en las que se organizan las diferentes funciones y relaciones individuales (González Rey, 2014a). De este modo, entender la identidad en un marco subjetivo conlleva ir más allá de la definición clásica sobre los fondos de identidad (Esteban-Guitart, 2014a). Entendemos los fondos de identidad subjetivados como producciones simbólico-emocionales singulares esenciales para la comprensión, expresión y definición que pueden ser conscientes o no, y que emergen en el flujo de sentidos y configuraciones subjetivas que tienen su origen en la cultura pero que van más allá de ella, mediante procesos auto-generativos, que están simultáneamente organizadas en las historias de vida de las personas.

Incidir en los fondos de identidad en el marco de la teoría de la subjetividad significa avanzar en procesos que propician la característica humana de la creatividad. De este modo, el aprendizaje creativo emerge mediante el desarrollo de los fondos de identidad subjetivados a partir de una forma cualitativamente diferente de aprendizaje, en que el aprendiz, en su condición subjetiva, personaliza la información, lleva el contenido a sus propias perspectivas y genera ideas que se mueven más allá de las que inicialmente se plantean (Almeida y Soares Muniz, 2019). Así, es necesario hacer emerger los sentidos y configuraciones subjetivas que tienen su origen en los contextos históricos, en relaciones sociales, conocimientos y en situaciones singulares de la trayectoria de vida del aprendiz para posibilitar, mediante procesos como la fantasía y la imaginación, una posición activa y generadora del 
niño que contribuya a organizar y subjetivar los nuevos conocimientos en sistemas a partir de sentidos y configuraciones nuevas (Mitjáns Martínez, 2013a).

La emergencia del conocimiento subjetivado en los aprendices, es un proceso de construcción progresiva que depende del valor de aquellas experiencias que sean subjetivadas por el alumno en base a procesos de aprendizaje sensibles con espacios socio-relacionales en donde el individuo está inserto y en los sistemas de actividad y comunicación en donde el individuo participa (Mitjáns Martínez, 2013a). La escuela, siendo uno de los espacios socio-relacionales fundamentales donde los niños y los jóvenes participan ha de favorecer espacios de actividades-comunicación que potencialmente puedan ser generadores de nuevos sentidos subjetivos constitutivos de aprendizajes creativos (Mitjáns Martínez, 2013a).

Albertina Mitjáns (2013a) destaca dos acciones específicas para avanzar en el desarrollo de aprendizajes creativos. La primera de ellas consiste en la promoción del desarrollo de recursos subjetivos por parte del aprendiz. Es decir, se busca favorecer prácticas educativas que propicien que el aprendiz se convierta en sujeto de su propio aprendizaje y que el aprendizaje que desempeñe se establezca como configuración subjetiva generadora de nuevos sentidos subjetivos en el proceso de aprender. En segundo término, para favorecer el desarrollo de recursos subjetivos en el aprendizaje, es fundamental la personalización de dicho proceso de enseñanza. Es decir, es necesario que los docentes conozcan a los alumnos en toda su complejidad a partir de sus experiencias de vida y en sus sistemas de relaciones; aspectos determinantes en la subjetividad del aprendiz. Sólo así, será posible promover acciones educativas que sean sensibles a la singularidad estudiantil en los procesos de desarrollo y subjetivación. En definitiva, siendo sensibles a la emergencia de los aprendices como sujetos (González Rey y Mitjáns Martínez, 2017b).

El aprendizaje creativo se convierte en prospectivo a través de procesos de producción en donde el individuo se convierte en sujeto mediante la relación y el diálogo característicos del escenario de aprender. De este modo, si entendemos el conocimiento vehiculado por sentidos subjetivos de unidades simbólico-emocionales significativas para el alumno, más que por funciones cognitivas, es necesario la utilización de estrategias en el aula que favorezcan el diálogo, la producción discursiva y prácticas que favorezcan sentidos subjetivos por parte de los estudiantes (González Rey, 2007a). En ese proceso es esencial la cualidad del diálogo que se establece entre interlocutores de la dinámica relacional en la escuela. La dialogicidad se entiende como «un proceso que envuelve siempre los individuos como agentes activos en el diálogo, lo que los caracteriza como producción subjetiva y no como definición ontológica que reduce los procesos humanos a realidades dialógicas, omitiendo la presencia de la subjetividad como producción diferenciada de los sujetos o agentes de diálogo» (Mitjáns Martínez y González Rey, 2017, p. 87).

La dialogicidad se constituye como un proceso natural de producción de conocimiento que reconoce al sujeto o a los agentes del diálogo en su singularidad sin 
dejarse llevar por la reducción de los procesos humanos a realidades dialógicas. La singularidad se convierte de este modo en un recurso legítimo para construir conocimiento mediante procesos que van más allá de la comprensión de la investigación cualitativa clásica en doónde las evidencias y los resultados se convierten en el fin último del proceso. De este modo, la metodología constructivo-interpretativa (González Rey, 2007b; González Rey y Mitjáns Martínez, 2019), estrechamente ligada con la epistemología y la teoría, considera el diálogo con los estudiantes como momentos de expresión para fomentar la emergencia de nuevos sentidos subjetivos que están relacionados con sus experiencias diarias en la escuela y otros espacios relacionales (González Rey, 2014b; González Rey y Mitjáns Martínez, 2016). En este proceso, el conocimiento emerge como una construcción teorética que avanza en construcciones hipotéticas que desarrolla el investigador educativo. Esta construcción tiene un marcado carácter histórico y cultural ya que se conforma a partir de las características subjetivas de los sujetos concretos. A través de discusiones y reflexiones, el investigador educativo ejerce un rol activo y analítico, pero a su vez, las producciones que desarrollan los participantes no están bajo ningún control que encorsete o limite las producciones subjetivas de estos. La investigación educativa no se basa en un sistema fijo de premisas establecidas a priori, sino que avanza por vías y opciones alternativas a partir de reflexiones, dudas e ideas (González Rey y Mitjáns Martínez, 2019).

Lo novedoso de la metodología constructivo-interpretativa en las prácticas educativas se resume en lo siguiente. En un primer momento, hace posible reconocer la singularidad de cada alumno mediante el diálogo, la comunicación y la relación con el otro superando de este modo la tradición metodológica en psicológica, tanto cuantitativa como cualitativa, de generalización de los procesos y problemas que son experimentados por los estudiantes en los contextos escolares. En segundo lugar, entiende que para aproximarnos a las dinámicas relacionales que envuelven el aprendizaje escolar en la diversidad de lo singular, se precisa una nueva forma de aproximación al fenómeno que avance en la construcción de las diferentes configuraciones subjetivas de los alumnos (Almeida y Soares Muniz, 2019). Para ello, es necesaria dejar de lado la aplicación de metodologías e instrumentos aislados. Es decir, los instrumentos metodológicos tales como narrativas, observación participativa, complementos de frases, dibujos y fotografías e incluso metodologías autobiográficas extendidas (Esteban-Guitart, 2012; 2016), se usan desde este enfoque para promocionar diálogos constructivo-interpretativos avanzando hacia la inteligibilidad compleja del fenómeno educativo.

Después de haber revisado la literatura sobre la teoría de la subjetividad con relación al aprendizaje (Mitjáns Martínez y González Rey, 2017; Almeida y Soares Muniz, 2019; Coelho y Tacca, 2019; Santos Bezerra y Rossato, 2019; Patino y Caires de Souza, 2019), hemos detectado algunas experiencias que podemos usar para ilustrar cómo las instituciones escolares pueden usar fondos de identidad bajo un 
marco subjetivo con beneficios pedagógicos. Estos ejemplos proporcionan una ilustración fértil de cómo podemos llevar a cabo la educación prospectiva, poniendo la identidad subjetivada de los estudiantes en el centro de cualquier acto educativo. Esto enriquece los procesos de aprendizaje creativos porque enfatizan el proceso de creación, no incrustado en los recursos distribuidos que las personas usan para dar sentido y significado, sino en unidades simbólico-emocionales autogeneradas por el aprendiz que posibilita las emergencias de sentidos y configuraciones subjetivas, abriendo de este modo nuevas vías de subjetivación eminentemente genuinas y singulares.

\section{ESTRATEgIAS Y RECURSOS PARA USAR PEDAGógICAMENTE}

En particular, se pone el foco en dos experiencias que ilustran cómo la escuela conecta con las experiencias de vida de los estudiantes a partir de sus producciones subjetivas más allá de la institución escolar. Se destaca, así, cómo los sentidos y configuraciones subjetivas viajan a través de entornos sociales reales e imaginarios en donde las producciones subjetivas de los estudiantes se han dado lugar a través de procesos de diálogo y comunicación con el otro mediante procesos constructivointerpretativos.

Los dos ejemplos que discutimos aquí representan sugerencias de organización de las prácticas escolares para facilitar el aprendizaje basado en las identidades de los estudiantes como unidades simbólico-emocionales y auto-generativas. Es decir, las configuraciones subjetivas iniciales con relación a las identidades de los aprendices se transformaron en configuraciones subjetivas de desarrollo. Los ejemplos analizados en este artículo son representativos como experiencias educativas basadas en el aprendizaje prospectivo (Kozulin, 1998) y describen formas innovadoras de fomentar aprendizajes creativos, centrándose en las producciones subjetivas y singulares de los aprendices.

Los estudios de caso llevado a cabo por Almeida y Muniz (2019) han tenido como pretensión desarrollar experiencias de aprendizaje en que el aprendiz, en su condición subjetiva, hiciera emerger procesos creativos en situaciones de aprendizaje a partir de complejas y dinámicas redes de producciones subjetivas. El componente metodológico es comprensivo-interpretativo, y la metodología se desenvuelve a partir de momentos en que la producción subjetiva de los participantes emerge durante diálogos y actividades a través de la implicación espontánea de los participantes. Es en la interacción con el investigador educativo y en el uso de diferentes instrumentos, así como mediante observaciones en contextos escolares y no formales, que se comienzan a elaborar producciones teoréticas constructivo-interpretativas sobre las configuraciones subjetivas del aprendizaje en ambos participantes. Finalmente, ambos estudios de caso se desarrollan bajo el marco de la teoría de la subjetividad y lo que a continuación se expone es una relectura de los mismos con base en los fondos de identidad subjetivados. 
DAVID SUBERO Y MOISÉS ESTEBAN-GUITART

MÁS ALLÁ DEL APRENDIZAJE ESCOLAR: EL ROL DE LA SUBJETIVIDAD

EN EL ENFOQUE DE LOS FONDOS DE IDENTIDAD

\subsection{El caso de Gabriel}

Gabriel es un niño de nueve años que hace tercero de primaria, en que sus fondos de identidad subjetivados iniciales fueron el dibujo y la escritura. El dibujo y la lectura son expresiones de configuraciones subjetivas. Inicialmente, estos fondos estaban asociados con un aspecto lúdico del aprendizaje (Almeida y Soares Muniz, 2019), es decir, como relación personal, espontánea, libre, investigativa y voluntaria del niño con el aprendizaje. En este espacio lúdico, Gabriel era capaz de escapar de la vida real a través de las simulaciones que le proporcionaban sus fondos identidad e ir más allá de la propia experiencia, creando sus propias reglas y un escenario imaginado. Estos fondos de identidad subjetivados emergieron en el proceso constructivo-interpretativo, así como a partir del uso de instrumentos tales como frases de rastreo - el niño completaba las frases que se le proporcionaban de forma que emergieran producciones subjetivas - , diarios de ideas — una libreta en blanco posibilitaba que el niño apuntara expresiones espontáneas en ella más allá de lo que es requerido en la escuela - y contando mi historia —uso de libros y dibujos para entender la historia de vida del niño-(Almeida y Muniz, 2019). Es a partir de la información que los instrumentos posibilitan, la escritura y el dibujo, que la atención de los investigadores educativos se centra en identificar cómo estos pueden ser un recurso de sentidos subjetivos en el proceso de aprender a leer y escribir. Entendemos de este modo, que la relación entre la acción de dibujar y las acciones de leer y escribir están configuradas en flujos de recursos subjetivos, que incluyen en la autonomía, la concentración, la euforia, así como en una actitud hacia la investigación por parte del niño que busca avanzar en niveles más altos de expresión. En el proceso constructivo-interpretativo iniciado con el niño, emerge la figura del padre como importantes sentidos subjetivos en la relación de Gabriel con el aprendizaje de la lectura y la escritura. El padre es un indiciador de sentido subjetivo del valor de éste para el niño en relación con la experiencia de aprendizaje. La figura subjetivada del padre se convierte en sentidos subjetivos importantes en la autodefinición de Gabriel como diseñador de letras alfabéticas, cosa que comportó cambios en las relaciones de éste con sus compañeros. Los dibujos que Gabriel realizaba en la escuela fueron reconocidos por sus compañeros y su profesor como valiosos. Al sentirse útil, emerge y se establece una configuración subjetiva a través de una trama de sentidos desarrollados en el espacio social escolar —es decir, subjetividad social - sobre las habilidades de Gabriel para dibujar. La subjetividad social de la escuela se traducía en configuraciones subjetivas singulares de Gabriel como elemento de socialización, seguridad y mejora de sus propias habilidades en el dibujo. A partir de estos sentidos y configuraciones subjetivas sobre el dibujo, Gabriel desarrolló unidades simbólico-emocionales autogeneradas, vehiculadas por la fantasía y la imaginación, en el establecimiento de un espacio social creativo en la clase en que no solamente enseñaba sus dibujos sino que ayudaba a sus compañeros a aprender a dibujar como él. La profesora, reconociendo el interés de Gabriel por 
el dibujo, facilitó la extensión de las prácticas y aprendizajes creativos que se empezaban a generar en el aula de tal modo que empezó a incluir espacios de dibujo en las actividades de escritura con carácter formalizado. La actividad se consolidó de tal modo que, "dibujando cartas" se convirtió en una actividad en que la escritura se mezclaba con el dibujo de tal manera que se daba un giro a la escritura como era entendida de forma tradicional en la asignatura estableciendo así la escritura como rastreo de cartas y elaboración de nuevas formas de letras que las acompañaban.

\subsection{El caso de Camila}

Camila es una joven de dieciocho años que participó en un proyecto educativo no formal de difusión científica de duración anual llamado "Salud en las escuelas» (Almeida, 2015). Los fondos de identidad subjetivados iniciales fueron su postura crítica sobre temas sociales, asociados con las esferas políticas y morales, así como la lucha por la justicia social. Estos fondos de identidad subjetivados emergieron en el proceso constructivo-interpretativo mediante entrevistas individuales y semiestructuradas sobre la vida y la historia escolar de la joven. Además, se usaron instrumentos tales como frases completas, elaboración de ensayos, así como documentos de análisis producidos por la joven a través de un portafolio, sus cuadernos escolares y sus registros. También se realizaron entrevistas a los monitores del proyecto y a dos profesores de la escuela (Almeida y Muniz, 2019). Los intereses de Camila por la justicia social, la política, el racismo, la discriminación de género y la lucha contra la pobreza estaban presentes en sus publicaciones en las redes sociales. Además, una de las aficiones más relevantes de la joven era escuchar y cantar música "rap» con carga política y social. A lo largo del proyecto las configuraciones subjetivas de la joven en base al aprendizaje creativo se configuraron de la siguiente forma. En un primer momento, la joven intervino a lo largo de las actividades y discusiones del proyecto presentando argumentos que iban en contra del sentido común de la discusión sorprendiendo con fuertes actitudes críticas y de confrontación hacia los problemas políticos y sociales. Su intervención en el grupo, caracterizada por una postura audaz y alternativa tanto en su reflexión crítica sobre los temas a abordar, así como su oposición a la subjetividad social dominante, representada por el monitor de la actividad, planteaba nuevas posibilidades de afrontar la situación y nuevas perspectivas en asociar ideas y generar hipótesis explicativas sobre los temas de discusión. Su postura comprometida en las discusiones de política y sociedad hizo que se comprometiera con el proyecto y desarrollara producciones subjetivas autogenerativas propias que hicieron enriquecer y amplificar el resultado del aprendizaje. Un ejemplo de ello fue la personalización del concepto «salud». En el contexto del proyecto, Camila históricamente configuró sentidos subjetivos que estuvieron relacionados con su postura crítica sobre temas sociales, asociados con sus valores morales y luchas por la justicia social que posibilitaron nuevas producciones subjetivas sobre su preocupación por el éxito en la escuela y el reconocimiento social, 
por el proyecto de grupo, y sus actividades en políticas escolares. Es decir, las configuraciones subjetivas del aprendizaje trascendieron el proyecto y emergieron también en la escuela. El origen de su postura crítica hacia los temas seleccionados fueron más bien las aficiones, motivaciones e intereses de Camila que no las prácticas o experiencias que la joven hubiera desarrollado con sus padres o en el contexto familiar. Sin embargo, el ser de clase social baja, mujer, afrobrasileña, el contexto de extrarradio en dónde vivía y la inquietud por las mejoras en relación a las clases y personas desfavorecidas tuvieron un impacto importante en la subjetividad de Camila. Estas nuevas producciones subjetivas fueron posibles a través de cuestionamientos genuinos, mostrando seguridad y actitudes audaces a la hora de producir nuevos conocimientos y procesos de socialización. La forma en que Camila subjetivó socialmente los espacios escolares y los del proyecto educativo, a partir de los sentidos subjetivos de expectativas morales y políticas que ya tenía configurados, posibilitaron nuevas vías de subjetivación de las acciones de aprendizaje de forma singular y personalizada al posicionarse como sujeto creativo.

\section{CONCLUSIONES}

Empezamos identificando la necesidad de una nueva comprensión del aprendizaje en un contexto global interconectado, en que cada vez es más necesario movilizar habilidades de pensamiento crítico, innovador y creativo que serán prioritarios en la economía global: el aprendizaje prospectivo (Kozulin, 1998). Acorde con este enfoque, el aprendizaje se entiende como aquél en que los aprendices han de ser capaces de afrontar problemas todavía inexistentes a partir de un proceso creativo, el proceso de "autoría».

Desde nuestro punto de vista el aprendizaje creativo (Mitjáns Martínez, 2013a; Mitjáns Martínez y González Rey; 2017) así como los fondos de identidad bajo la teoría de la subjetividad de corte histórico-cultural ejemplifican aprendizajes prospectivos que enriquecen y tienen en cuenta en última instancia la producción subjetiva por parte del aprendiz de forma singular. El investigador educativo, posibilita procesos constructivo-interpretativos de diálogo y comunicación con el otro que propicia la emergencia del sujeto en los procesos de aprendizaje abriendo nuevas vías de subjetivación en el aprendiz. De este modo, el educador debe considerar los fondos de identidad subjetivados de los estudiantes como configuraciones subjetivas iniciales de los aprendices que ayudan a organizar el aprendizaje en entornos escolares; entendiendo estos como espacios de emergencia de subjetividades sociales (González Rey y Mitjáns Martínez, 2017b).

Para nosotros, un tema central de los proyectos y estrategias pedagógicas que se describen en el artículo es lo que González Rey y Mitjáns Martínez (2017a) llaman sentidos y configuraciones subjetivas y su importancia para el aprendizaje. Es decir, se sugiere que cualquier pedagogía inclusiva tiene que reconocer las experiencias y las vivencias de los estudiantes a partir de unidades subjetivas histórica y culturalmente 
configuradas (descritos arriba como uno de los indicadores de pedagogía efectiva para el CREDE), o lo que nosotros nos referimos como fondos de identidad en el marco de la subjetividad.

El reto educativo consiste en la promoción de espacios de aprendizaje escolar donde los estudiantes produzcan nuevos sentidos subjetivos a partir de la producción de subjetividades, tanto individuales como sociales, que estén estrechamente relacionadas con la imaginación y la fantasía (Mitjáns Martínez, 2013a). En el caso de Gabriel y de Camila, sus sentidos subjetivos históricamente relacionados con sus dibujos y sus inquietudes por la justicia y transformación social, respectivamente, estuvieron asociados con la libertad para expresar sus ideas. Tales producciones fueron conectadas con sus sentidos actuales que estaban asociados con los deseos en Gabriel de una escritura original con dibujos y letras características y originales y, en el caso de Camila, con sus sentidos de trascender el proyecto de salud con un trasfondo crítico, socialmente justo y reivindicativo más allá del espacio no formal, como en la escuela y en otros espacios comunitarios.

De este modo, los aprendices integraron sentidos subjetivos en diversos espacios de aprendizaje dentro y fuera de la escuela. Estos sentidos subjetivos estuvieron relacionados principalmente con espacios sociales tales como la escuela en el caso de Gabriel, o espacios no formales en el caso de Camila con el proyecto de "Salud». Sin embargo, dichos sentidos subjetivos no pudieron estar disociados de los sentidos subjetivos generados en otros espacios sociales y sistemas relacionales mediante procesos de subjetividad social. Un ejemplo de ello fue la influencia de la familia (padre) en el caso de Gabriel, o bien la familia, raza, género, clase social y económica en el caso de Camila. En ambos casos, la confluencia de sentidos subjetivos individuales y sociales, generó nuevos sentidos subjetivos que favoreció el desarrollo de configuraciones subjetivas que abrieron espacios a diferentes actitudes y desempeños en la escuela.

En este sentido, el profesor puede hacer emerger los fondos de identidad subjetivados de los estudiantes, es decir, sentidos subjetivos históricamente configurados que están relacionados con una expresión, definición, valoración singular por parte del aprendiz y favorecer la emergencia de nuevos sentidos subjetivos que trasciendan las ideas ya existentes y avancen en los procesos de creación y de autoría mediante procesos reflexivos guiados por la metodología constructivo-interpretativa, así como instrumentos que abran vías de inteligibilidad a través del diálogo y la comunicación con el otro. Entendemos que hacer posible la emergencia de estos fondos de identidad subjetivados (en el caso de Gabriel la relación de su padre con el dibujo facilitó nuevas producciones del niño en la escuela) y a su vez, reconocer esos sentidos subjetivos, puede favorecer el establecimiento de configuraciones subjetivas que animen el desarrollo y emergencia de nuevas producciones por parte del sujeto (desarrollo en el espacio de clase de aprendizajes creativos y de autoría por parte de Gabriel vinculando la escritura y el dibujo de las letras con sus compañeros). 
La complejidad de los procesos de aprendizaje como producción subjetiva solicitan por parte del docente tener un rol flexible y creativo a la hora de posibilitar las prácticas escolares. El hecho es que, si entendemos la complejidad de la subjetividad en toda su totalidad, ésta no permite pensar en una influencia directa entre lo planeado por el docente y el efecto resultante en el aula por parte de los aprendices. Es decir, "el docente puede tener un sentido y una intencionalidad, mientras que el alumno, en función de sus configuraciones subjetivas - en las que participan otras influencias y otros espacios relacionales- genera otros sentidos subjetivos» (Mitjáns Martínez, 2013b, p. 433). Si nos ponemos a pensar, son innumerables las veces que el profesor genera conocimiento teórico que está relacionado con lo que él considera mejor para el alumno. Este "mejor para el otro» (Goulart, 2019) es una premisa racional que de hecho, pueden conducir a la imposición como docente. Muchas veces, estas vías de subjetivación pueden estar relacionadas con resistencias, sufrimientos o dificultades que luego pueden derivar en dificultades de aprendizaje, fracaso o abandono escolar (Santos-Bezerra y Rossato, 2019). De este modo, el docente ha de abrir espacios posibles para la subjetivación creando espacios propicios basados en la dialogicidad que hagan viable la producción de sentidos subjetivos para favorecer el aprendizaje creativo. Los procesos constructivointerpretativo demuestran que la emergencia de la creatividad en el aprendizaje ocurre a través de momentos de expresión dinámicos y complejos de la subjetividad de los aprendices como individuos concretos y productores de sentidos y configuraciones subjetivas (Oliveira Campolina y Mitjáns Martínez, 2016; 2019).

Para los educadores, ser sensible a los fondos de identidad subjetivados de los estudiantes comporta dinamizar el aprendizaje en un sentido creativo. Implementar actividades educativas eminentemente creativas posibilita el desarrollo de sentidos subjetivos que presentan desafíos, fantasías, que colocan a los aprendices en situaciones de producir, problematizar y pensar. El investigador educativo ejerce de este modo un rol que no es el de transmisor de conocimientos, sino quien propone desafíos a los alumnos, que estimulan su imaginación, su pensamiento y su carácter generador (Mitjáns Martínez, 2013a). De este modo, los fondos de identidad subjetivados pueden poner al aprendiz en el centro del aprendizaje a través de su emergencia como sujeto, a través de producciones subjetivas singulares basadas en la historia de vida del sujeto.

Para concluir, en las sociedades contemporáneas la mayoría de las personas han de desarrollar competencias que les permitan trabajar y personalizar la información, llevar el contenido a sus propias perspectivas y generar ideas que se mueven más allá de las que inicialmente se plantean de forma creativa, en lugar de asimilar y reproducir pasivamente una tradición cultural sólida e incuestionable. Los contextos educativos actuales deben enseñar a los jóvenes a ampliar el potencial de aprendizaje de los alumnos y las habilidades necesarias para ser genuinos, ir más allá de los problemas actuales y plantear propuestas innovadoras guiadas por 
la imaginación y la fantasía. Creemos que combinar los fondos de identidad desde un marco subjetivo puede promover mayores cotas de aprendizaje creativo en los alumnos, hecho que facilitará mayores cotas de continuidad en los aprendizajes de los alumnos en un contexto de aprendizaje interconectado. Avanzar en esta tipología de aprendizaje se sugiere relevante para enfrentar los desafíos de aprendizaje y los cambios sistémicos que afectan a las sociedades y culturas en la economía global.

\section{REFERENCIAS BIBLIOGRÁFICAS}

Almeida, P. (2015). A aprendizagem criativa em contextos não-formais: caracterização e processos subjetivos constitutivos. Ph.D. Thesis, Universidade de Brasilia, Brasília, DF, Brasil.

Almeida, P. y Soares Muniz, L. (2019). Subjective Configurations of Action and the Emergence of Creative Learning. En F. González Rey, A. Mitjáns Martínez, y D. M. Goulart (eds.), Subjectivity within Cultural-Historical approach (pp. 21-60). Singapore: Springer. https:// doi.org/10.1007/978-981-13-3155-8.

Araya, D. y Michael, A. (2010). Education in the Creative Economy. New York, NY: Peter Lang.

Azevedo, F. S. (2013). The tailored practice of hobbies and its implications for the design of interest-driven learning environments. Journal of the Learning Sciences, 22(3), 462-510. https://doi.org/10.1080/10508406.2012.730082.

CREDE (2004). CREDE History. Center for Research on Education, Diversity, and Excellence, University of Hawai'i. Recuperado de: http://manoa.hawaii.edu/coe/credenational/ crede-history/ (Consultado el 15/04/2019).

Erstad, O., y Sefton-Green, J. (2012). Identity, community, and learning lives in the digital age. Cambridge-New York: Cambridge University Press.

Esteban-Guitart, M. (2012). La multimetodología Autobiográfica Extendida y los fondos de identidad. Revista de Ciencias Sociales. 18 (4), 587-600.

Esteban-Guitart, M. (2016). Funds of identity. New York, NY: Cambridge University Press. https://doi.org/10.1017/CBO9781316544884.

Esteban-Guitart, M., y Moll, L. C. (2014a). Funds of identity: A new concept based on the funds of knowledge approach. Culture \& Psychology, 20(1), 31-48. https://doi. org/10.1177/1354067X13515934 .

Esteban-Guitart, M., y Moll, L. C. (2014b). Lived experience, funds of identity and education. Culture \& Psychology, 20(1), 70-81. https://doi.org/10.1177/1354067X13515940 .

Esteban-Guitart, M. y Vila, I. (2013). Experiencias en educación inclusiva. Vinculación escuela, familia y comunidad. Barcelona: Horsori.

Esteban-Guitart, M., Subero, D., y Brito-Rivera, L. F. (2015). La Incorporación de los Fondos de Identidad en un Centro Abierto. El Proyecto FICAB. Multidisciplinary Journal of Educational Research, 5(1), 55-81. http://dx.doi.org/10.447/remie.2015.03.

González Rey, F. L. (2007a). Psicoterapia, subjetividade e pós-modernidade: uma aproximação histórico cultural. São Paulo: Thompson Learning. https://doi.org/10.1590/S010279722009000200019.

González Rey, F. (2007b). Los procesos de construcción de la información. Mc Graw Hill: México. 
DAVID SUBERO Y MOISÉS ESTEBAN-GUITART

MÁS ALLÁ DEL APRENDIZAJE ESCOLAR: EL ROL DE LA SUBJETIVIDAD EN EL ENFOQUE DE LOS FONDOS DE IDENTIDAD

González Rey, F. (2011). A re-examination of defining moments in Vygotsky's work and their implications for his continuing legacy. Mind, Culture and Activity, 18(3), 257-275. https:// doi.org/10.1080/10749030903338517.

González Rey, F. (2014a). Human Motivation in Question: Discussing Emotions, Motives, and Subjectivity from a Cultural-Historical Standpoint. Journal for the Theory of Social Behaviour 45(4). https://doi.org/10.1111/jtsb.12073.

González Rey, F. (2014b). Ideias e modelos teóricos na pesquisa construtivo-interpretativa En A. Mitjáns Martínez, M. S. Neubern, y V. D. Mori (Eds.), Subjetividade Contemporânea: discussões epistemológicas e metodológicas (pp. 13-34). Alínea: Campinas.

González Rey, F. L. (2018). Subjectivity in debate: Some reconstructed philosophical premises to advance its discussion in psychology. Journal for The Theory of Social Behaviour. 1-23. https://doi.org/10.1111/jtsb.12200.

González Rey, F., y Mitjáns Martinez, A. (2016). Una epistemologia para el estudio de la subjetividad: sus implicaciones metodológicas. Perspectivas. Individuo y Sociedad, 15, 5-16. https://doi.org/10.5027/PSICOPERSPECTIVAS-VOL15- ISSUE1-FULLTEXT-667.

González Rey, F. y Mitjáns Martínez, M. (2017a). El desarrollo de la subjetividad: una alternativa frente a las teorías del desarrollo psíquico. Papeles de Trabajo sobre Cultura, Educación y Desarrollo Humano, 13(2), 3-20.

González Rey, F. L., y Mitjáns-Martínez, A. (2017b). Subjetividade. Teoria, epistemología e método [Subjectivity. Theory, epistemology and method]. Campinas: Alínea.

González Rey, F. y Mitjáns Martínez, A. (2019). The Constructive-Interpretative Methodological Approach: Orienting Research and Practice on the Basis of Subjectivity. En F. González Rey, A. Mitjáns Martínez, y D. M. Goulart (eds.), Subjectivity within Cultural-Historical approach (pp. 37-60). Singapore: Springer. https://doi.org/10.1007/978-981-13-3155-8.

González, N., Moll, L. C., y Amanti, C. (Eds.). (2005). Funds of knowledge: Theorizing practices in households, communities and classrooms. Mahwah, NJ: Lawrence Erlbaum Associates.

Goulart, D. (2019) Subject and Subjectivity in Psychotherapy: A Case Study. En F. González Rey, A. Mitjáns y D.M. Goulart (Eds.) (2019). Subjectivity within cultural-bistorical approach. Singapore: Springer.

Ito, M., Gutiérrez, K., Livingstone, S., Penuel, B., Rhodes, J., Salen, K., Schor, J., Sefton-Green, J, y Watkins, S. C. (2013). Connected Learning. An Agenda for Research and Design. Irvine, CA: Digital Media and Learning Research Hub.

Kozulin, A. (1998). Psychological tools. A sociocultural approach to education. Cambridge, MA: Harvard University Press.

Livingstone, S., y Sefton-Green, J., (2016). The Class: Living and Learning in the Digital Age. New York: New York University Press.

Luning, R. I., Wyatt, T., y Im, S. (2011). Classroom Observation Rubric: Development and Validation of a Measure of Effective Pedagogy. CREDE Hawai'i Project. Honolulu, HI: University of Hawai'i.

Madeira-Coelho, C. y Tacca, M. C. (2019). Subjectivity in a Cultural-Historical Perspective: New Theoretical Insight on Educational Processes and Practices. En F. González Rey, A. Mitjáns Martínez, y D. M. Goulart (eds.), Subjectivity within Cultural-Historical approach (pp. 21-60). Singapore: Springer. https://doi.org/10.1007/978-981-13-3155-8 . 
Mitjáns Martínez, A. (2013a). Aprendizaje Creativo: Desafíos para la práctica pedagógica. Revista CS, 11, 311-341.

Mitjáns Martínez, A. (2013b). Creatividad y Subjetividad: Su expresión en el contexto escolar. Revista Diversitas-Perspectivas en psicología, 9(2), 427-434.

Mitjáns Martínez, A., y González Rey, F. (2017). Psicologia, Educaçao e Aprendizagem Escolar. Cortez Editora: Brasil.

Moll, L. C. (1997). Vygotsky, la educación y la cultura en acción. En A. Álavarez (Ed.), Hacia un currículum cultural. La vigencia de Vygotski en la educación (pp. 39-53). Madrid: Fundación Infancia y Aprendizaje.

Moll, L. (2014). L. S. Vygotsky and education. New York, NY: Routledge.

National Research Council. (2012). Education for Life and Work: Developing Transferable Knowledge and Skills in the 21st Century. Washington, D.C. Recuperado de:_http:// www7.national-academies.org/BOTA/Education_for_Life_and_Work_report_brief.pdf (Consultado el 15/04/2019).

Oliveira Campolina, L. y Mitjáns Martínez, A. (2016). The configuration of social subjectivity in an innovative educational institution in Brazil. International Research in Early Childhood Education, 7 (1), 182-200. https://doi.org/10.4225/03/58100484489c4.

Oliveira Campolina, L. y Mitjáns Martínez, A. (2019). The Role of Subjectivity in the Process of School Innovation. En F. González Rey, A. Mitjáns Martínez, y D. M. Goulart (eds.), Subjectivity within Cultural-Historical approach (pp. 21-60). Singapore: Springer. https:// doi.org/10.1007/978-981-13-3155-8 .

Patino, J. F., y Caires de Souza, E. (2019). Discussing Subjectivity in Undergraduate and Graduate Education. En F. González Rey, A. Mitjáns Martínez, y D. M. Goulart (eds.), Subjectivity within Cultural-Historical approach (pp. 21-60). Singapore: Springer. https:// doi.org/10.1007/978-981-13-3155-8 .

Penuel, W. R., y Wertsch, J. V. (1995). Vygotsky and identity formation: A sociocultural approach. Educational Psychologist, 30(2), 83-92. http://dx.doi.org/10.1207/s15326985ep3002_5.

Rios-Aguilar, C., Marquez-Kiyama, J., Gravitt, M., y Moll, L. (2011). Funds of knowledge for the por and forms of capital for the rich? A capital approach to examining funds of knowledge. Theory and Research in Education, 9, 163-175. https://doi.org/10.1177/1477878511409776.

Saz, A., Engel, A., y e Coll, C. (2016). Introducing a personal learning environment in higher education. An analysis of connectivity. Digital Education Review 29, 1-14. https://doi. org/10.1344/der.2016.29.1-14.

Santos Bezerra, M. y Rossato, M. (2019). Relational Dynamics in Overcoming School Learning Difficulties. En F. González Rey, A. Mitjáns Martínez, y D. M. Goulart (eds.), Subjectivity within Cultural-Historical approach (pp. 21-60). Singapore: Springer. https://doi. org/10.1007/978-981-13-3155-8 .

Subero, D. (En prensa). Fondos de identidad y subjetividad: búsqueda de nuevas vías y alternativas para un diálogo productivo. Estudios de Psicología.

Subero, D., Vila, I. y Esteban-Guitart, M. (2015). Some Contemporary Forms of the Funds of Knowledge Approach. Developing Culturally Responsive Pedagogy for Social Justice. International Journal of Educational Psychology, 4(1), 33-53. https://doi.org/10.4471/ ijep.2015.02. 
Subero, D., Vujasinović, E. y Esteban-Guitart, M. (2017). Mobilising funds of identity in and out of school, Cambridge Journal of Education, 47(2), 247-263. https://doi.org/10.108 0/0305764X.2016.1148116.

Talizina, N. (2000). Manual de Psicología Pedagógica. Mexico: Editorial Universitarioa Potosina.

Talizina, N., Solovieva, Y. y Quintanar-Rojas, L. (2010) La aproximación de la actividad en psicología y su relación con el enfoque histórico-cultural de L. S. Vigotsky. Novedades Educativas, 230, 4-8.

Williams, R. (1999). The behavioral perspective in contemporary education. The Teacher Educator, 35(2), 44-60. https://doi.org/10.1080/08878739909555225. 